\title{
Correlation between Ankle Brachial Index with Coronary Artery Disease and Gender
}

Suresh Yerra ${ }^{1}$ Shravan Kumar ${ }^{1} \quad$ Indrani Garre ${ }^{1} \quad$ Achukatla Kumar $^{1}$
Address for correspondence Suresh Yerra, MD, (DM), Cardiology 2nd-year Resident, Department of Cardiology, Nizam's Institute of Medical Sciences, Punjagutta 500082, Hyderabad, Telangana, India (e-mail: yerrasuresh3@gmail.com).

Indian J Cardiovasc Dis Women-WINCARS 2018;3:231-236

\begin{abstract}
Keywords

- ankle brachial index

- atherosclerosis

- coronary artery disease

Background Ankle brachial index ( $A B I)$ has been shown to be a specific and sensitive for the diagnosis of peripheral artery disease (PAD). Both PAD and coronary artery disease (CAD) are caused by atherosclerosis, which is a narrowing of the arteries due to the build-up of plaque and fatty material inside the lumen. The aim of our study is to find out the relation between $A B I$ and coronary angiographic findings with cardiovascular risk factors and gender in patients admitted in a tertiary care center with a provisional diagnosis of CAD.

Methods It is a cross-sectional observational study, in which patients admitted with suspected CAD are included. In this study, patient characteristics such as demographics, family history, past history of CAD, and other comorbidities were included, and also the risk factors for atherosclerosis such as hypertension, diabetes, dyslipidemia, and smoking history were enquired by using a predetermined proforma. In all study patients, $A B I$ was measured prior to coronary artery angiogram (CAG). $A B I$ $\leq 0.9$ was considered as $\mathrm{PAD}$, and $\mathrm{ABI}$ between 0.9 and 1.4 was considered as normal. Then, all patients included in the study underwent CAG. The results of the ABI reports were compared with the angiographic findings and atherosclerotic risk factors.

Results In this study, 90 patients were included who were admitted for CAG. Twenty-two patients have $A B I \leq 1.09$ among them, 9 patients have no $C A D$, and 13 patients have $C A D$ on $C A G$. Sixty-eight patients have $A B I \geq 1.10$, with 28 patients having no $C A D$ and 40 patients having $C A D$. There is difference in the right and left mean $A B I$ with right $A B I$ less than left $A B I$, and it is statistically significant $(p=0.014)$. By binary logistic regression, the determinants of $C A D$ are left ventricle ejection fraction (EF) and age, and $A B I$ was not a predictor. In our study, out of 90 patients, only 1 had $A B I<0.9(1.1 \%)$. Therefore, it is not worth doing $A B I$ in all patients undergoing CAG to rule out peripheral vascular disease (PVD). This is true in male and female patients and even in the diabetics. In comparing male and female $A B I$, there is no significance between $A B I$ in both the sexes.

Conclusion This study findings concluded that $A B I$ is not a useful method in assessing the risk factors and the severity of CAD in suspected patients. Only left ventricle EF and age were the predictors of the presence of $C A D$, and $A B I$ was not a predictor. This study have shown the difference in the right and left mean $A B I$ with right $A B I$ less than left $A B I$, which is an important finding. This study has also shown the similar prevalence of $A B I$ in both sexes. However, to make more accurate results on $A B I$ (right vs. left), we should plan studies with larger sample size in future.
\end{abstract}

(C)2018 Women in Cardiology and Related Sciences
License terms

$($ () (1) $\odot \circledast$ 


\section{Introduction}

The ratio of systolic blood pressure at the ankle and the systolic pressure at the arm is defined as the ankle brachial index (ABI). ABI has been shown to be a sensitive and specific index for the diagnosis of peripheral artery disease (PAD). ${ }^{1}$ Both PAD and coronary artery disease (CAD) are caused by atherosclerosis, which is a narrowing of the arteries due to the built-up of plaque and fatty material inside the lumen. Atherosclerosis is the most common cause of mortality and morbidity worldwide. ${ }^{2}$ The aim of our study is to find out the relation between $A B I$ and coronary angiographic findings with cardiovascular risk factors and gender in patients admitted in a tertiary care center with a provisional diagnosis of CAD.

\section{Methods}

It is a cross-sectional observational study, in which patients admitted with suspected CAD are included. Inclusion criteria include all the patients who were admitted for coronary artery angiogram (CAG) between January and May 2018. Patients with acute coronary syndromes, PAD, abnormal high $A B I$ (ratio > 1.4) causing false high due to calcification, and who were unwilling to participate in the study were excluded from the study. In this study, patient characteristics such as demographics, family history, past history of CAD, and other comorbidities were included, and also the risk factors for atherosclerosis such as hypertension, diabetes, dyslipidemia, and smoking history were enquired by using a predetermined proforma.

Ankle brachial index was calculated by measuring the systolic pressure at the right brachial artery (denominator) and the systolic pressure at the right dorsalis pedis artery (numerator) by the manual auscultatory method; ratio of the both gives right $A B I$. The same method is repeated on the left limbs obtaining the left ABI. Measurements were done according to the standard recommendations for the calculation of ABI by the American Heart Association (AHA). ABI was measured in all studied patients prior to CAG. ABI value $\leq 0.9$ was considered as $P A D$, and $A B I$ value $>0.9$ was considered as normal. Then, all patients included in this study underwent CAG.

\section{Definition of Coronary Artery Disease}

A disease in which there is a narrowing or blockage of the coronary arteries usually due to atherosclerosis (buildup of plaque and fatty material in the lumen of the coronary arteries). Severity of CAD is estimated by coronary angiography. Stenoses are defined as minimal if the narrowing is $<50 \%$, moderate between 50 and $70 \%$, and severe or significant for a diameter reduction of $\geq 70 \%$.

\section{Statistical Analysis}

The angiographic reports and the questionnaire proforma were compared with ABI results. Data were analyzed by MINITAB 17 (Minitab Inc.), using $t$-test, binary logistic regression, equivalence test, and chi-square test. Two sample $t$-test was used to compare mean and standard deviation of the right and left $A B I$ and the gender difference in $A B I$. Binary logistic regression was used to compare CAD severity with age, gender, ejection fraction (EF), ABI, comorbidities, and risk factors. Equivalence test with paired data was used to compare right and left $\mathrm{ABI}$.

\section{Results}

In this study, 90 patients were included who were admitted for CAG during the study period.

Among the total 90 study population, 22 (24.4\%) are female patients and $68(75.6 \%)$ are male patients (-Fig. 1). Majority of the study population are between 40 and 70 years of age contributing to $78(87 \%)$. Most of them are between 50 and 60 years of age contributing to $36(40 \%)$ ( - Fig. 2).

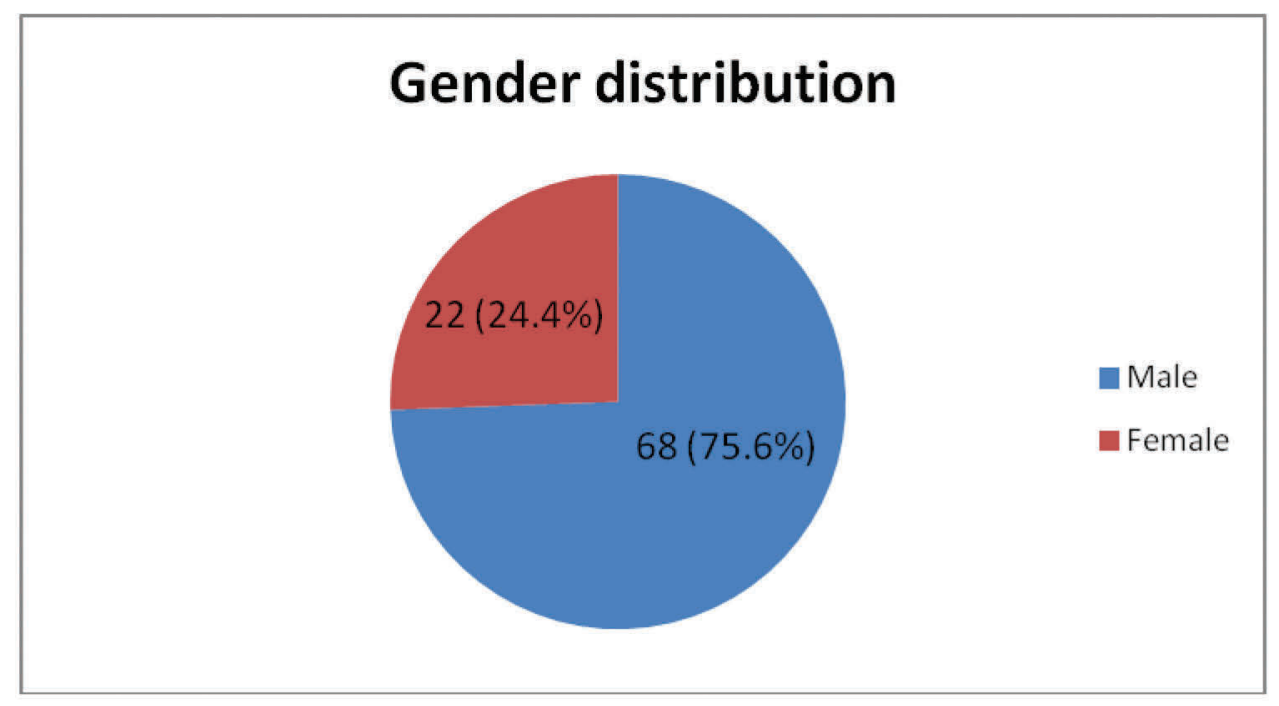

Fig. 1 Pie diagram showing the gender distribution in the study population. 
Among the study population, only one patient has ABI $<0.9$, so we have subdivided the study population into two groups, one group with $\mathrm{ABI}$ between $<1.09$ and second group with $A B I \geq 1.10$. In our study, we have done this to find out whether there is any difference in the extent of CAD, and gender exists between the two groups ( - Fig. 3 ).

Twenty-two patients have $\mathrm{ABI} \leq 1.09$, among them nine patients have no CAD and 13 patients have CAD on CAG. Sixty-eight patients have $A B I \geq 1.10$, with 28 (41.2\%) patients having no CAD and 40 (58.8\%) having CAD (-Fig. 4).

Box plot of right and left mean $A B I(-$ Fig. 5) shows difference in the right and left mean $A B I$ with right $A B I$
( mean $=1.1130)$ less than left $\mathrm{ABI}($ mean $=1.1338)$, and it is statistically significant $(p=0.014$ ).

There is no difference between the male and female patients with regard to right $A B I$, even though there is minor difference in the data, but it is not statistically significant $(p=0.446)$. Same is applicable even for the left ABI showing no difference in male and female patients $(p=0.594)(-$ Table $\mathbf{1})$.

Binary logistic test was done to known the determinants of CAD. Among them only left ventricle EF and age were the predictors of the presence of $C A D$, and $A B I$ was not a predictor. In our study, out of 90 patients, only 1 had $\mathrm{ABI}<0.9(1.1 \%)$.

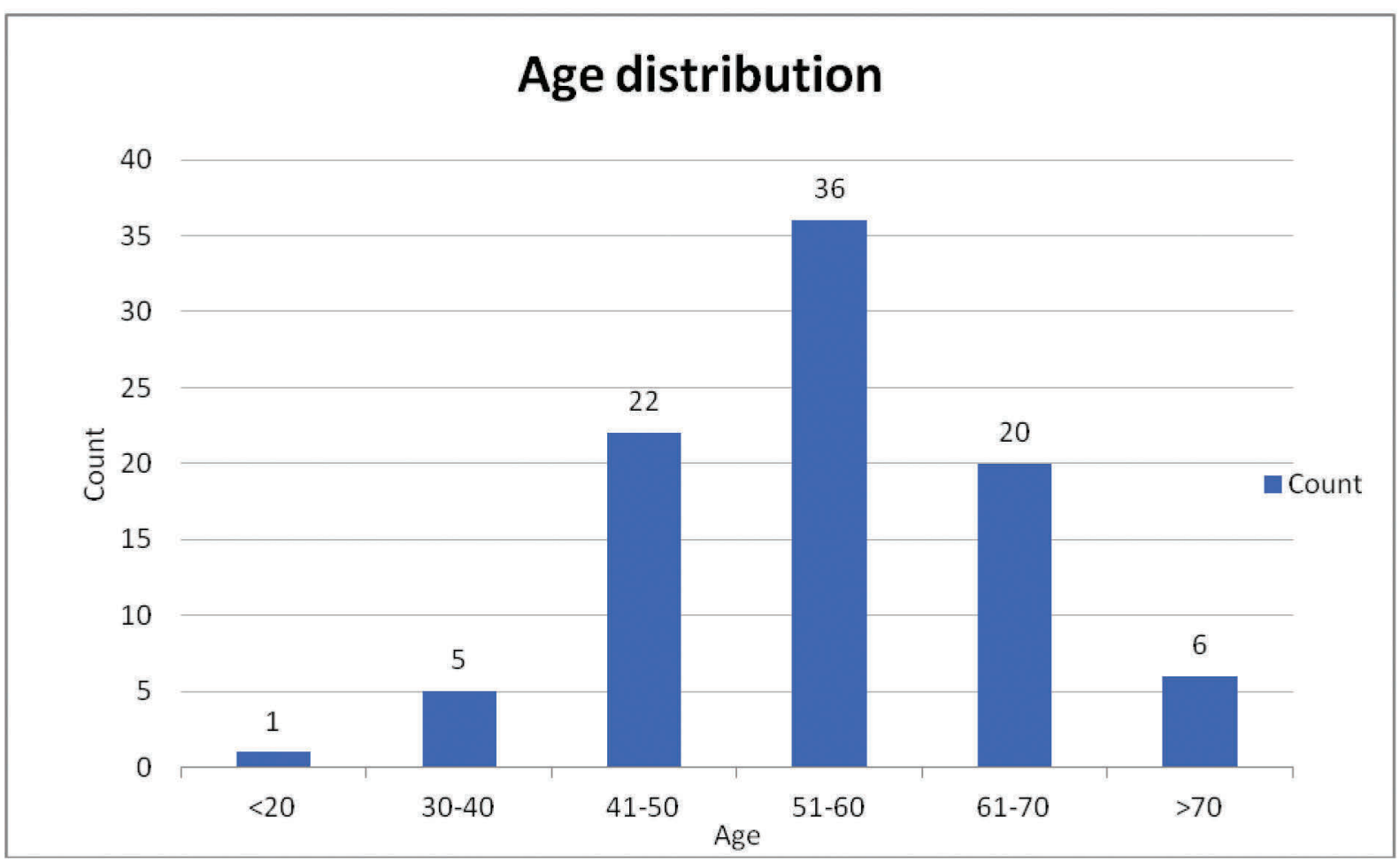

Fig. 2 Bar diagram showing the age distribution.

\section{Two groups based on $A B I$}

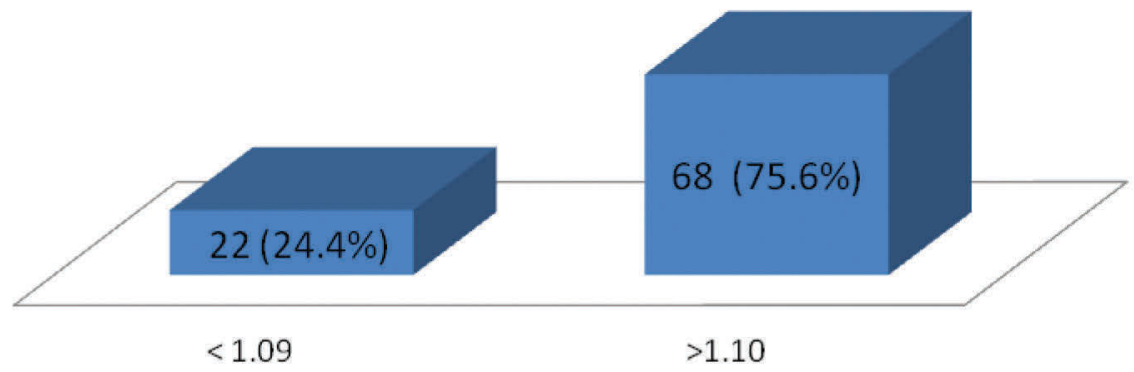

Fig. 3 Bar diagram of ankle brachial index (ABI) in both groups. 


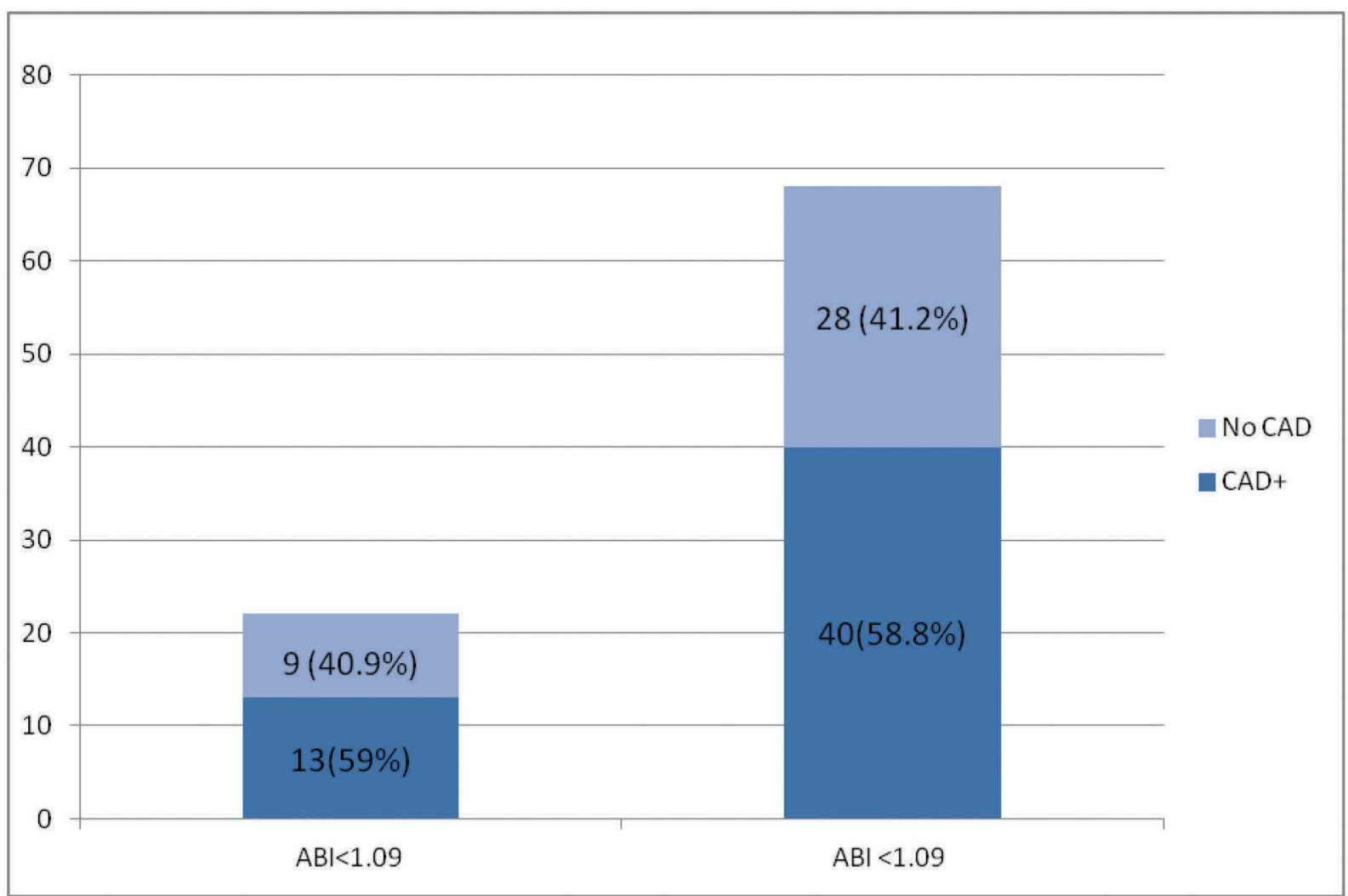

Fig. 4 Stack bar diagram showing comparison between the two groups with CAD+ or no CAD. ABI, ankle brachial index; CAD, coronary artery disease.

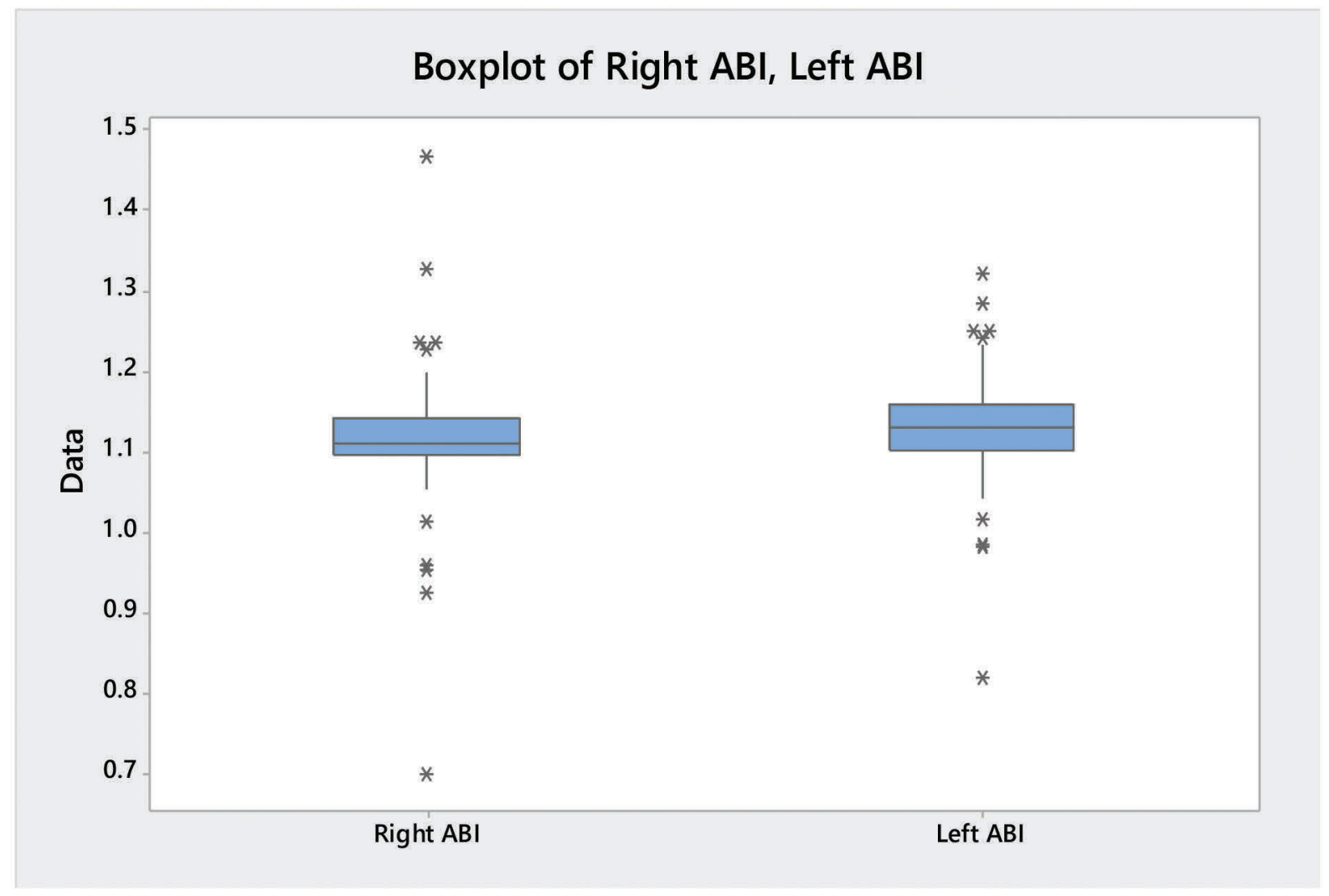

Fig. 5 Box plot of right and left ankle brachial index (ABI). 
Table 1 Binary logistic regression results to know the determinants of CAD

\begin{tabular}{|l|l|l|}
\hline Source & Chi-square & $p$ Value \\
\hline Age & 3.96 & $\mathbf{0 . 0 4 6}$ \\
\hline EF\% & $\mathbf{4 . 3 0}$ & $\mathbf{0 . 0 3 8}$ \\
\hline Right ABI & 0.04 & 0.843 \\
\hline Left ABI & 1.15 & 0.284 \\
\hline Sex & 2.55 & 0.111 \\
\hline HTN & 0.42 & 0.518 \\
\hline DM & 0.11 & 0.745 \\
\hline Smoking & 1.06 & 0.302 \\
\hline Alcohol & 0.09 & 0.759 \\
\hline Previous CAD & 0.01 & 0.904 \\
\hline CSA/USA & 0.02 & 0.889 \\
\hline
\end{tabular}

Abbreviations: $A B I$, ankle brachial index; $C A D$, coronary artery disease; CSA, central sleep apnea; DM, diabetes mellitus; EF, ejection fraction; HTN, hypertension; USA, unstable angina.

Note: Values in bold have a significant $p$-value $<0.05$.

Therefore, it is not worth doing ABI in all patients undergoing coronary angiography to rule out peripheral vascular disease (PVD). This is true in male and patients and even in the diabetics. In comparing male and female $\mathrm{ABI}$, there is no significance between $\mathrm{ABI}$ in both the sexes.

\section{Discussion}

Ankle brachial index ratios of 0.90 to 1.40 are considered normal for adults, and ratios $<0.9$ indicate that PAD is present. Lower readings $<0.7$ suggest that the disease is severe and that patients might develop digital gangrenes or ulcers in the lower limbs. Ratios > 1.4 suggest a noncompressible calcified vessel, which happens in diabetic or elderly patients due to fibrotic or calcified vessels. The aim of our study was to find the correlation between the ABI with CAD and gender. Among the study population, 25\% were female patients and the rest $75 \%$ were male patients. Among them only one patient had $\mathrm{ABI}<0.9$. Therefore, we have divided the study population into two groups with $\mathrm{ABI}<1.09$ and $>1.10$ to find out whether any difference exists between the two groups and whether $A B I$ cutoff could be increased to increase the sensitivity and use $A B I$ as a screening test for CAD.

Multiple large population-based studies done by Criqui et al and in the Framingham study concluded that ABI is a marker of atherosclerosis and has a good correlation with cardiovascular disease (CVD). ${ }^{3-5}$ In asymptomatic patients who are having intermediate-risk factors for CVD, ABI measurement is advised as a part of primary prevention and risk stratification of CVD by Greenland et al., 6,7

Vogt et al reported in their study that the mortality rates from atherosclerotic coronary diseases are doubled with every 0.5 unit decrease in $A B I$ value. In patients with an $A B I$ value $<0.5,10$-year mortality was $37 \%$ compared with $17 \%$ in patients with $\mathrm{ABI}>0.9(p=0.0039){ }^{8}$
In the Framingham offspring study by Murabito et al, CAD prevalence was $30 \%$ in patients with $\mathrm{ABI}<0.9$ compared with $10 \%$ with $\mathrm{ABI}>1.0$, with a significant $p$ value $(p=0.001) .{ }^{9}$

Regarding gender difference of $\mathrm{ABI}$, there are different results in various studies. Our study results were in similar line with the study done by Taylor-Piliae et al, which showed the ABI prevalence was similar with no gender difference, ${ }^{10}$ and these findings could be explained by the high rates of CAD in men. However, Sadrzadeh Rafie et al in California studied the relation of gender differences on the prevalence of PAD in patients who were referred for elective CAG. This study showed that even though the prevalence and severity of CAD is lower in women, prevalence of PAD is more among them. ${ }^{11}$ Papamichael et al and Ramos et al have studied 165 patients referred for elective CAG, among them 15 to $20 \%$ had $A B I$ value $\leq 0.9$, which was significantly higher in male patients compared with female patients. ${ }^{10,12}$ Therefore for more conclusive results, we need more studies in future with a larger sample size.

In our study, only left ventricle EF and age were the predictors of the presence of $C A D$, and $A B I$ was not a predictor. However, in a study done in Taiwan, Chang et al studied the correlation of $A B I$ in patients undergoing elective CAG to predict the severity of complex and diffuse coronary artery lesions. They have indicated that among the risk factors such as smoking, hypertension and diabetes were significantly more prevalent in $\mathrm{ABI}+$ patients (ABI value $\leq 0.9$ ). Furthermore, compared with the control group with $A B I>0.9$, the $\mathrm{ABI}+$ patients had more stenotic and critical coronary lesions, which were difficult for intervention. Hence, they have recommended this well-established, simple, and inexpensive test for diagnosing PAD, and also useful for predicting complex and diffuse lesion subtypes that would be useful in interventions and during follow-up period. ${ }^{13}$

A systematic review by Anand and Doobay "determined the sensitivity and specificity of $\mathrm{ABI}$ in the prediction of future cardiovascular events." It was concluded that though $\mathrm{ABI} \leq 0.9$ is highly specific but not sensitive in prediction of CVD, and because of its simple assessment, it is considered as a useful tool for prediction of cardiovascular events risk. ${ }^{14}$

Ankle brachial index test is a simple test that is noninvasive and inexpensive and is widely used clinically as a screening test. However, ABI has its own limitations. Elderly and diabetic patients with calcified and fibrotic vessels may have a high values due to differential calcification leading to noncompressible vessel in the ankle compared with upper limbs. Duplex ultrasound is also a noninvasive test that shows blood flow in the arteries, but it requires an experienced radiologist to achieve useful images. Computed tomography (CT) or magnetic resonance angiography can also help image the blood vessels with limitations such as cost, contrast, and radiation side effects.

Latest studies have shown that an ABI value of $>1.4$ is considered as a marker of noncompressible, calcified arteries, and it could also helps in prediction of an increased risk of cardiovascular events. ${ }^{15}$ 


\section{Conclusion}

This study findings concluded that $\mathrm{ABI}$ is not a useful method in assessing the risk factors and the severity of CAD in suspected high-risk patients. Only left ventricle EF and age were the predictors of the presence of CAD, and ABI was not a predictor. This study has shown the difference in the right and left mean $A B I$ with right $A B I$ less than left $A B I$, which is an important finding. This study has also shown the similar prevalence of $\mathrm{ABI}$ in both sexes. However, to make more accurate results on $\mathrm{ABI}$ (right vs. left), we should plan studies with larger sample size in future.

Interpretation of this study: Actual aim of the study (primary objective) is to see where ABI can detect CAD along with the severity of $C A D$, which was proved that routine $A B I$ in patients who were coming to CAG is not useful to predict the CAD. We found an interesting finding that there is difference in mean right and left $\mathrm{ABI}$ with right $\mathrm{ABI}$ less than left, and it is a bystander observation. To imply this result to general population, we may require separate ABI normality curves; we should plan further studies with large sample sizes of the general population.

\section{Conflict of Interest}

None.

\section{Limitations}

- It is a single-centered trail with a limited study population admitted for CAG from January to May 2018.

- It is a cross-sectional study, and follow-up of the patients was not done in this study.

\section{References}

1 Dachun X, Yawei X, Dayi H, et al. Sensitivity and specificity of the $\mathrm{ABI}$ to diagnose peripheral artery disease (PAD): a structured review. Vasc Med 2010;15(5):361-369

2 Caro J, Proskorovsky I, Ishak KJ, Migliaccio-Walle K. The morbidity and mortality following a diagnosis of the PAD: long-term follow-up of a large database. BMC Cardiovasc Disord 2005;5:14

3 Criqui MH, Langer RD, Fronek A, et al. Mortality over a period of 10 years in patients with peripheral arterial disease. $\mathrm{N}$ Engl J Med 1992;326(6):381-386
4 Murabito JM, Larson MG, Evans JC, Wilson PW, Nieto K, Levy D. Framingham Study. The ABI in the elderly and risk of stroke, coronary disease and death. Arch Intern Med 2003;63:1939-1942

5 Newmann AB, Shemanski L, Manolio TA, et al. ABI as a predictor of cardiovascular disease and mortality in the Cardiovascular Health Study. Arterioscler Thromb Vasc Bio 1999;19:538545 PubMed

6 Greenland P, Abrams J, Aurigemma GP, et al; Writing Group. Prevention Conference V: beyond secondary prevention: identifying the high-risk patient for primary prevention: noninvasive tests of atherosclerotic burden: Writing Group III. Circulation 2000;101(1):E16-E22

7 Greenland P, Smith SC Jr, Grundy SM. Improving coronary heart disease risk assessment in asymptomatic people: role of traditional risk factors and noninvasive cardiovascular tests. Circulation 2001;104(15):1863-1867

8 Vogt MT, McKenna M, Anderson SJ, Wolfson SK, Kuller LH. The relationship between ankle-arm index and mortality in older men and women. J Am Geriatr Soc 1993;41(5):523-530

9 Murabito JM, Larson MG, Levy D, Wilson PW, Evans JC, Nieto K. Prevalence and clinical correlates of PAD in the Framingham offspring study. Am Heart J 2002;143:961-965

10 Taylor-Piliae RE, Fair JM, Varady AN, et al. Ankle brachial index screening in asymptomatic older adults. Am Heart J 2011;161(5):979-8511

11 Sadrzadeh Rafie AH, Stefanick ML, Sims ST, et al. Sex differences in the prevalence of peripheral artery disease in patients undergoing coronary catheterization. Vasc Med 2010;15(6):443-450

12 Papamichael CM, Lekakis JP, Stamatelopoulos KS, et al. Ankle-brachial index as a predictor of the extent of coronary atherosclerosis and cardiovascular events in patients with coronary artery disease. Am J Cardiol 2000;86(6):615-618

13 Chang ST, Cheng HW, Chung CM, et al. Ankle-arm index as a predictor of lesion morphology and risk classification for CAD undergoing coronary angioplasty. Int J Cardiol 2006;113(3):385-390

14 Doobay AV, Anand SS. Sensitivity and specificity of the ankle-brachial index to predict future cardiovascular outcomes: a systematic review. Arterioscler Thromb Vasc Biol 2005;25(7):1463-1469

15 Espinola-Klein C, Rupprecht HJ, Bickel C, et al; AtheroGene Investigators. Different calculations of ankle-brachial index and their impact on cardiovascular risk prediction. Circulation 2008;118(9):961-967 